\title{
THE MEANING OF MONO AND EPI \\ IN SOME FAMILIAR CATEGORIES
}

\author{
W. Burgess \\ (received May 1, 1965)
}

1. Introduction. This expository note was prompted by some questions asked by Professor P. Hilton during his lectures "Catégories non-abéliennes" at the University of MontréaI, JuIy 1964.

The descriptions of set functions as one to one and as onto can be characterized in terms of set function composition. A set function is one to one iff it has the left cancellation property, that is, $f \cdot g=f \cdot h$ implies $g=h$. A set function is onto iff it has the right cancellation property, that is, $g \cdot f=h \cdot f$ implies $g=h$. The cancellation properties can be expressed in any category so we make the

DEFINITION 1 . In a category $\zeta$ a map $f$ is mono iff it has the left cancellation property; and, $f$ is called epi iff it has the right cancellation property.

The purpose of this note is to characterize mono and epi in some specific categories. In what follows, a full subcategory $B$ of $\zeta$ is a subcategory of $\zeta$ such that $\operatorname{Hom}_{\beta} \frac{(A, B)=\operatorname{Hom}}{\zeta^{(A, B)}}$ for all $A, B \in \mathcal{B}$. A concrete category is one whose objects are sets and whose maps are a subclass of the class of set functions.

Conversations with Professors W. Kuyk, J. Lambek and B. Rattray have been most helpful.

2. Mono. A sufficient condition for maps in concrete categories to be mono is trivial.

Canad. Math. BuIl. vol. 8, no. 6, 1965. 
PROPOSITION 2. In every concrete category one to one implies mono.

Proof. Let $f: A \rightarrow B$ be one to one and $g, h: C \rightarrow A$ be such that $f \cdot g=f \cdot h$. If $g(x) \neq h(x)$ for some $x \in C$ then $(f \cdot g)(x)=f(g(x)) \neq f(h(x))=(f \cdot h)(x)$ by definition of one to one. Q.E. D.

THEOREM 3. In the following concrete categories mono is equivalent to one to one:

(1) any full subcategory of the category of topological spaces (maps are continuous functions);

(2) the category of based topological spaces (an object is a topological space with distinguished point and maps are continuous functions which map base point onto base point), and any full subcategory which contains a two point space with the discrete topology;

(3) any full subcategory of the category of groups (with group homomorphisms as maps) which contains with any group all its normal subgroups;

(4) any full subcategory of the category of rings (with ring homomorphisms as maps) which contains with any ring all its ideals;

(5) the category of infinite groups as a full subcategory of the category of groups.

Proof. In each case we show that if a map is not one to one it is not mono. For (1), assume $f: S_{1} \rightarrow S_{2}$ is such that $f(s)=f\left(s^{\prime}\right)$ and $s \neq s^{\prime}$. Let $T$ be any topological space from the category and let $\mathrm{g}, \mathrm{h}: \mathrm{T} \rightarrow \mathrm{S}_{1}$ be the constant maps onto $\mathrm{s}$ and $s^{\prime}$ respectively.

(2) If $f:\left(X, x_{0}\right) \rightarrow\left(Y, y_{0}\right)$ and $f(x)=f\left(x^{\prime}\right), x \neq x^{\prime}$ let $\left(T, t_{0}\right)=\left\{t_{0}, t_{1}\right\}$ with the discrete topology and then define $g, h:\left(T, t_{0}\right) \rightarrow\left(X, x_{0}\right)$ by $g\left(t_{1}\right)=x, h\left(t_{1}\right)=x^{\prime}$. 
(3) If $f: G_{1} \rightarrow G_{2}$ is not one to one, $\{1\} \neq \operatorname{ker} f=K$ is an object of the category. Let $g, h: K \rightarrow G_{1}$ be the inclusion map and the trivial map of $K$ into $G_{1}$ respectively. Similarly for (4).

(5) This part is included because the technique used here can be readily generalized. Let $f: G_{1} \rightarrow G_{2}$ be not one to one and let $C$ denote the infinite cyclic group on the generator $c$, then define $g, h: C \rightarrow G_{1}$ by $g(c)=k, 1 \neq k \in$ ker $f$, and $h(c)=1$.

Q. E. D.

We now proceed with the generalization mentioned above. An algebraic system is called equationally defined if its structure is defined by a family of finite operations and a family of identities involving these operations. For example, a group is a set with two operations (multiplication and inverse) which satisfy certain identities. Consider a particular equationally defined class of algebraic systems called $\sigma$-systems, which are defined by the operations $f_{1}, f_{2}, \ldots$ (not necessarily countable) such that $f_{\beta}$ is an $n(\beta)$-ary operation, $n(\beta)$ finite, and some set of identities involving these operations.

Given a set of symbols $\left\{x_{1}, x_{2}, \ldots\right\}$ a free $\sigma$-system may be constructed with these symbols as generators (see, for example [1]). Intuitively speaking, the process is to consider all expressions built from the generators and the operations and then make only the identifications suggested by the given set of identities. The resulting set of equivalence classes can then be considered as a $\sigma$-system.

THEOREM 4. Let $\zeta$ be the category of all $\sigma$-systems with $\sigma$-homomorphisms as maps (set functions which preserve all the operations) then mono is equivalent to one to one.

Proof. Let $A, B$ be $\sigma$-systems and $f: A \rightarrow B$ a $\sigma$-homomorphism then if $f(a)=f\left(a^{\prime}\right)$ and $a \neq a^{\prime}$ we can proceed as follows. Denote by $F$ the free $\sigma$-system on one generator $x$. Define $g, h: F \rightarrow A$ by $g(x)=a, h(x)=a^{\prime}$. Since $g$ and $h$ are defined on the generator of $F$ they can be extended, uniquely, to $\sigma$-homomorphisms. Q.E.D. 
Examples of equationally defined systems are groups, rings and $R$-modules, $R$ a fixed ring. In the case of $R$-modules, each element from $R$ - is considered as a unary operation in the module. Examples of algebraic systems which are not equationally defined are fields and torsion-free Abelian groups.

The class of small categories (categories whose class of objects is a set) is known to form a category with functors as maps.

THEOREM 5. In the category of small categories, a map (functor) is mono iff it is one to one as a function on objects and on maps.

Proof. The sufficiency of the condition is clear. In what follows $A, B, \mathscr{C}, \ldots$ are categories; $A, B, C, \ldots$ objects in the categories; $a, b, c, \ldots$ maps of the categories; and, the letters $f, g$, and $h$ are reserved for functors. Let $f: A \rightarrow B$ be such that $A \neq A^{\prime}$ but $f(A)=f\left(A^{\prime}\right)$ then let $\mathscr{C}$ be any small category and $g, h: \mathscr{\zeta} \rightarrow A$ be defined by $g(C)=A$ for each $C$ in $\mathscr{C}$ and $g(c)={ }_{A}$, the identity map on $A$, for each $c$ in $\mathscr{C}$. Similarly, $h(C)=A^{\prime}$ and $h(c)={ }_{A^{\prime}}$. Thus, $f \cdot g=f \cdot h$ and $g \neq h$ implying that $f$ is not mono.

If $f$ is assumed to be mono and if $f(a)=f\left(a^{\prime}\right)$ then $a$ and $a^{\prime}$ have the same range and domain since we have shown that $f$ is one to one on the objects. Let $a, a^{\prime}: A \rightarrow A^{\prime}$ and let $\mathscr{C}$ be a category with two objects $\mathrm{C}$ and $C^{\prime}$ and only one non-identity map $c: C \rightarrow C^{\prime}$. Define $g, h: \mathscr{C} \rightarrow A$ by $g(C)=h(C)=A$, $g\left(C^{\prime}\right)=h\left(C^{\prime}\right)=A^{\prime}, g(c)=a$ and $h(c)=a^{\prime}$. It is clear that $g$ and $h$ are functors and this contradicts the assumption that $f$ is mono unless $a=a^{\prime}$. Q.E. D.

Let $A$ be an Abelian category ([2]) and $\mathcal{B}$ a small category, then the class of functors from $B$ to $A$ is a category with natural transformations as maps. Moreover, this category, $\not^{B}$, is a category with kernels. If $\sigma: F \rightarrow G$ is a map in $A^{B}$ then $\sigma(B): F(B) \rightarrow G(B)$ is a map in $A$ for each $B$ in $B$. Let $K(B)$ be the domain of $k(B)=\operatorname{ker} \sigma(B)$. Then for any $\mathrm{b}: \mathrm{B}_{1} \rightarrow \mathrm{B}_{2}$ in $\mathcal{B}$ we have the diagram 


$$
\begin{aligned}
& k\left(B_{1}\right) \quad \sigma\left(B_{1}\right) \quad \text { and the square on } \\
& \mathrm{K}\left(\mathrm{B}_{1}\right) \rightarrow \mathrm{F}\left(\mathrm{B}_{1}\right) \rightarrow \quad \mathrm{G}\left(\mathrm{B}_{1}\right) \quad \begin{array}{l}
\text { the right commutes. } \\
\text { We wish to complete }
\end{array}
\end{aligned}
$$

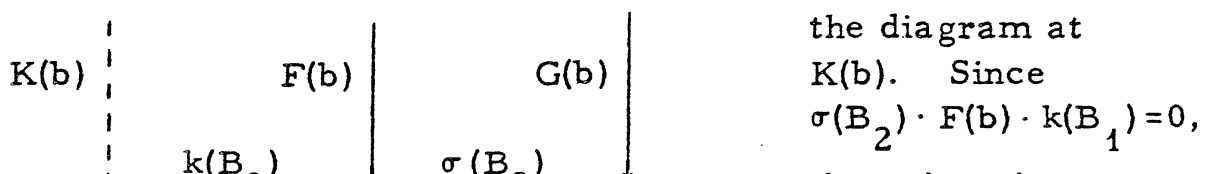

$$
\begin{aligned}
& \text { there is unique } \\
& \mathrm{K}\left(\mathrm{B}_{2}\right) \rightarrow \mathrm{F}\left(\mathrm{B}_{2}\right) \rightarrow \mathrm{G}\left(\mathrm{B}_{2}\right)^{\cdot} \quad \text { completion by the } \\
& \text { definition of a } \\
& \text { kernel in } A \text {. }
\end{aligned}
$$

By the uniqueness, $K$ is a functor, and since the diagram commutes, $k$ is a natural transformation. It is easy to see that $k$ is a kernel of $\sigma$.

Now, in a category with kernels a map has zero kernel if it is mono since if $f$ is mono, $f \cdot \operatorname{ker} f=f \cdot 0=0$ and so ker $\mathrm{f}=0$. If the category is Abelian, the converse is true since ker $f=0$ implies that if $f \cdot g=f \cdot h$ then $f \cdot(g-h)=0$ so $h-g=0$ and $h=g$.

PROPOSITION 6. If $A$ is Abelian and $B$ is small then a map $\sigma$ in $A^{\beta}$ is mono iff $\sigma(B)$ is mono for each $B$ in $\mathcal{B}$.

Proof. Clearly $\sigma(B)$ mono for each $B$ in $\beta$ implies $\sigma$ is mono. Conversely, if $\sigma$ is mono then ker $\sigma=0$ so $\operatorname{ker} \sigma(B)=0$ for each B. Q.E.D.

The following artificial example shows that there exist concrete categories where the notions of mono and one to one do not coincide. Let $\mathcal{G}$ be the category with objects $S_{1}=\left\{x_{1}, x_{2}, x_{3}\right\}$ and $S_{2}=\left\{y_{1}, y_{2}\right\}$ and maps $\sigma_{1}: S_{1} \rightarrow S_{1}$ where $\sigma_{1}\left(x_{i}\right)=x_{1}, \sigma_{2}: S_{1} \rightarrow S_{2}$ where $\sigma_{2}\left(x_{1}\right)=\sigma_{2}\left(x_{2}\right)=y_{1}$ and $\sigma_{2}\left(x_{3}\right)=y_{2}, \sigma_{2} \cdot \sigma_{1}$, and the two identity maps. Both $\sigma_{2}$ and $\sigma_{2} \cdot \sigma_{1}$ are mono but not one to one.

3. Epi. The problem of interpreting epi in concrete categories is somewhat more difficult. In fact there are familiar categories, such as the category of rings where the answer is apparently not known. Trivially, we have 
PROPOSITION 7. In a concrete category a map is epi if it is onto.

To describe epi in categories of topological spaces the following construction is useful. Let $U$ be a topological space and $A \subset U$ a subset. Form the disjoint union $U *=U_{1} \cup U_{2}$ where $U_{i}$ is a copy of $U$. Throughout, the subscript 1 indicates a point or set from the first copy of $U$ and the subscript 2 the same from the second copy. Thus, $a_{1}$ and $a_{2}$ or $S_{1}$ and $S_{2}$ are copies of the same point $a \in U$ or set $S \subset U$. Define an equivalence relation on $U *$ by $a_{1}=a_{2}$ for every $a \in A$ and call the space of equivalence classes with the quotient topology $U_{A}$.

The following two facts about this construction are easy to see. If $U$ is Hausdorff and $A$ is closed then $U_{A}$ is Hausdorff; and, if $U$ is $T_{1}$ and $A$ is open then $U_{A}$ is $T_{1}$.

In the sequel the maps of a category will not be specified if they are the obvious ones.

PROPOSITION 8. Let $\zeta$ be a full subcategory of the category of Hausdorff spaces such that if $U$ is in $\zeta$ and $A \subset U$ is closed then $U_{A}$ is in $\mathscr{b}$. Then, a map in $\mathscr{b}$ is epi iff its image is dense in its range.

Proof. Assume $f: S \rightarrow U$ is such that $f(S)$ is dense in $U$ and Ie $g, h: U \rightarrow U^{\prime}$ be distinct maps such that $g \cdot f=h$. $f$; but this contradicts the weIl known fact that a map with range a Hausdorff space is determined by its action on any dense subset.

If $f(S)$ is not dense in $U, A=\overline{f(S)} \neq U$ and, so, $U_{A}$ is not $A$. Let $g, h: U \rightarrow U_{A}$ be given by $g(x)=\bar{x}_{1}$ and $h(x)=\bar{x}_{2}$, where $\bar{x}_{i}$ is the equivalence class of $U_{A}$ with representative $x_{i}$. The two maps are distinct but coincide on A. Q.E.D. 
Proposition 8 does not hold in all full subcategories of the category of Hausdorff spaces, as the following example shows. Consider the space $R *$ which is the real line with the topology generated by the subbase consisting of the usual open sets plus the set of rationals. The space $R *$ is Hausdorff; the irrationals $I$ are closed and, so, not dense; the rationals $Q$ are open and dense. Let $\zeta$ be the full subcategory with objects $R *$ and $I$, as a subspace. Note that $I$ has the usual topology. We wish to show that the inclusion map of $I$ into $R *$ is epi, or that any two maps from $R *$ to either $R *$ or I which coincide on I must be equal. Assume $g, h: R * \rightarrow R *$ (or I) coincide on I. If $g(r) \neq h(r), r \in Q$, there is a neighbourhood $N=(a, b) \cap Q$ of $r$ such that $g(N) \cap h(N)=\emptyset$, and since closures are smaller in $R *$ than in the usual topology we may assume $\overline{g(N)} \cap \overline{h(N)}=\emptyset$. Now let $i \in I, a<i<b$ and $\left\{r_{i}\right\}$ a sequence from $N$ converging to $i$. A contradiction is apparent.

This is a convenient place to note that the notion of onto in the category of Hausdorff spaces can be expressed in categorical terms. The referee has pointed out that this was also noted in [5].

DEFINITION 9. ([3]) p. 32) An epi map $f$ is called pure iff $f=g \cdot h, \cdot g$ mono, implies that $h$ is epi.

THEOREM 10. In the category of Hausdorff spaces, a map is onto iff it is pure epi.

Proof. Let $f: A \rightarrow B, g: C \rightarrow B$ and $h: A \rightarrow C$. If $f$ is onto and $f=g \cdot h, g$ mono, then $g$ is one to one. Hence, $g \cdot h$ onto implies $h$ onto and so epi.

If $f$ is not onto, Iet $C=B$ as sets, and let $C$ have the topology generated by the open sets of $B$ and the sets $\{x\}$ for aII $x \notin f(A)$. Let $g$ be the set identity map which is mono and continuous since $C$ has a finer topology than $B$. Let $h(a)$ be the point $f(a)$ considered in $C$. The function $h$ is continuous since any open set $G$ of $C$ can be expressed in the form $G_{1} \cup G_{2}$ where $G_{1}$ is open in $B$ and $G_{2} \cap f(A)=0$.

Then $h^{-1}(G)=h^{-1}\left(G_{1}\right)=f^{-1}\left(G_{1}\right)$ which is open in $A$. The 
map $h$ is not epi since its image is a proper closed subset of $C$ and is, therefore, not dense. Q. E. D.

PROPOSITION 11. In the category (1) of all topological spaces and (2) the category of aII $T_{1}$-spaces, epi means onto.

Proof. If $f: S \rightarrow U$ is not onto, let $U^{\prime}=\{a, b\}$ with the trivial topology. Let $g, h: U \rightarrow U^{\prime}$ be given by $g(t)=a$ for all $t \in U$ and $h(t)=a$ if $t \in f(S)$ and $h(t)=b$ if $t \notin f(S)$.

(2) If $f: S \rightarrow U$ is not onto there is a point $x \notin f(S)$. Let $A$ be the complement of $\{x\}$, then $A$ is open. Form $U_{A}$ and let $g, h: U \rightarrow U_{A}$ be defined by $g(t)=\bar{t}_{1}$ and $h(t)=\bar{t}_{2}$. Q.E.D.

THEOREM 12. In the category (1) of Abelian groups and (2) of finite groups, epi means onto.

Proof. (1) Let $f: G_{1} \rightarrow G_{2}$ be not onto, then $f\left(G_{1}\right)$ is a proper subgroup of $G_{2}$. Let $g, h: G_{2} \rightarrow G_{2} / f\left(G_{1}\right)$ be the canonical and zero maps respectively.

(2) Let $f: A \rightarrow B$ be not onto. Let $H=f(A)$ and we have $B=x_{1} H \cup x_{2} H \cup \ldots U x_{m-1} H \cup H$ for some $x_{1}, \ldots, x_{m-1} \in B$ where $m=[\mathrm{B}: \mathrm{H}]$. Left multiplication of the se cosets by elements of $B$ gives a permutation of the cosets:

$$
\left(\begin{array}{c}
x_{i} H \\
b x_{i} H
\end{array}\right) \text {. Corresponding to } x_{i} H \text { the number } i \text { and to } H
$$
the number $m$, we have a representation of $B$ by a group of permutations

$$
\alpha(b)=\left(\begin{array}{llll}
1 & 2 & \ldots & m \\
i_{1} & i_{2} & & i_{m}
\end{array}\right)
$$

where $b x_{j} H=x_{i} H$. Also, if we correspond to $x_{i} H$ the number $i$ and to $H$ the number $m+1$, we get another, equivalent, representation 


$$
\beta(b)=\left(\begin{array}{lllll}
1 & 2 & \ldots & m-1 & m+1 \\
j_{1} & j_{2} & \cdots & j_{m-1} & j_{m+1}
\end{array}\right) \text {. }
$$

The two groups of permutations may be embedded in $S$ (permutation group on $m+1$ symbols) as follows:

$$
\begin{aligned}
& b \rightarrow \alpha(b) \rightarrow\left(\begin{array}{lllll}
1 & 2 & \ldots & m & m+1 \\
i_{1} & i_{2} & \ldots & i_{m} & m+1
\end{array}\right)=g(b) \\
& b \rightarrow \beta(b) \rightarrow\left(\begin{array}{lllll}
1 & 2 & \ldots & m & m+1 \\
j_{1} & j_{2} & \ldots & m & j_{m+1}
\end{array}\right)=h(b) .
\end{aligned}
$$

Since $b H=H$ iff $b \in H, b \in H$ implies $g(b)=h(b)$. Thus $g \cdot f=h \cdot f$ but $g \neq h$. Q. E. D.

THEOREM 13. In the categories (1) of aII groups and (2) of all torsion free groups epi means onto.

Proof. (1) Assume $\mathrm{f}: \mathrm{H} \rightarrow \mathrm{G}$ is not onto and form the free product of $G$ with itself with the subgroup $f(H)$ amalgamated ([6]), $K=G_{f(H)}^{*} G$. Define $g, h: G \rightarrow K$ by $g$ mapping $G$ onto the first copy of $G$ in $K$ and $h$ mapping $G$ onto the second: copy of $G$ in $K$. By construction of the amalgamated product, the se maps coincide on $f(H)$.

(2) The same proof holds in this case since the amalgamated free product of torsion-free groups is torsion-free. Q.E.D.

It is not always the case, in categories of algebraic systems, that epi means onto. We give two examples.

PROPOSITION 14. In the category of torsion-free Abelian groups $f: G_{1} \rightarrow G_{2}$ is epi iff $G_{2} / f\left(G_{1}\right)$ is a torsion group.

Proof. Let $G^{\prime}=G_{2} / f\left(G_{1}\right)$ and assume $G^{\prime}$ is not torsion, then if $T$ is the torsion subgroup of $G^{\prime}, G^{\prime} / T$ is a non-triviaI torsion-free group. Let $\mathrm{k}: \mathrm{G}_{2} \rightarrow \mathrm{G}^{\prime}$ be the canonical homomorphism and $k_{1}: G^{\prime} \rightarrow G^{\prime} / T$ the canonical homomorphism, $k_{2}: G^{\prime} \rightarrow G^{\prime} / T$ 
the zero homomorphism. Let. $g=k_{1} \cdot k, h=k_{2} \cdot k$ and then $g \neq h$ and $g \cdot f=h \cdot f=0$.

If $G^{\prime}$ is torsion, assume $g, h: G_{2} \rightarrow G_{3}$ are such that $g \cdot f=h \cdot f$ and $g \neq h$, then for some $a \in G_{2}, a \notin f\left(G_{1}\right)$, $g(a) \neq h(a)$. But $G^{\prime}$ is torsion so for some $n$, na $\in f\left(G_{1}\right)$ and $g(n a)=h(n a)=n g(a)=n h(a)$ and since $G_{3}$ is torsion-free, $g(a)=h(a)-a$ contradiction. Q. E. D.

Consider, now, the category of fields. This category is unusual since all maps are mono. If $f: K_{1} \rightarrow K_{2}$, this means that $K_{1}$ may be considered as a subfield of $K_{2}$. The statement that $g \cdot f=h \cdot f$ is equivalent to saying that $g$ and $h$ are isomorphisms of $\mathrm{K}_{2}$ over $\mathrm{K}_{1}$ (that is, they leave $\mathrm{K}_{1}$ pointwise invariant). Hence, restating the definition of epi in terms of fields we have

PROPOSITION 15. The embedding of $\mathrm{K}_{1}$ into $\mathrm{K}_{2}$ is epi iff $K_{2}$ has no non-trivial isomorphisms over $K_{1}$.

Proof. If $f$ is not epi clearly there are non-trivial isomorphisms. If $g$ and $h$ are non-trivial isomorphisms over $K_{1}$, $g\left(K_{2}\right)$ and $h\left(K_{2}\right)$ are extensions of $K_{1}$ and letting $L$ be an extension of $K_{1}$ containing both $g\left(K_{2}\right)$ and $h\left(K_{2}\right)$ we may consider $g$ and $h$ as having the same range and so $g \cdot f=h \cdot f$. Q. E. D.

As an example, consider $Z /(p), p$ prime, and let $K_{1}=[Z /(p)](t)$ where $t$ is transcendental over $Z /(p)$. The embedding of $K_{1}$ into $K_{1}(\sqrt[p]{t})$ is epi since $\sqrt[p]{t}$ has no conjugates over $K_{1}$ and so $K_{1}\left(\frac{P}{t}\right)$ admits no non-trivial isomorphisms.

In conclusion, using the notation of Proposition 6, we have its dual. 
PROPOSITION 16. If $A$ is Abelian and $B$ is small, then a map $\sigma$ in $\chi^{\beta}$ is epi iff $\sigma(B)$ is epi for each $B$ in $B$.

\section{REFERENCES}

1. G. Birkhoff, Lattice Theory. A.M.S., multilithed notes, 1963.

2. P. Freyd, Abelian Categories. Harper and Row, New York, 1964.

3. J.R. Isbell, Uniform Spaces. Math. Surveys, A. M. S., Providence, 1964.

4. J. L. Kelley, GeneraI Topology. Van Nostrand, New York, 1955.

5. H. Y. Kowalsky, Kategorien Topologischer Räume. Math. Z., 77 (249-272).

6. A. G. Kurosh, The Theory of Groups, vol. II. Chelsea, New York, 1960.

McGill University 